Tohoku J. Exp. Med., 2011, 224, 105-110

\title{
Suitability of Tartrate-Resistant Acid Phosphatase Type 5b as a Screening Marker for Bone Mineral Density in Community- Dwelling Elderly Individuals
}

\author{
Sumiko Irie, ${ }^{1}$ Naomi Hayashida, ${ }^{1}$ Tetsuko Shinkawa, ${ }^{1}$ Yasuyuki Taira, ${ }^{1}$ \\ Yui Sekitani, ${ }^{1}$ Seitaro Teraoka, ${ }^{1}$ Kanami Hashiguchi, ${ }^{1}$ Koji Yoshida, ${ }^{1}$ \\ Michiko Morishita ${ }^{1}$ and Noboru Takamura ${ }^{1}$ \\ ${ }^{1}$ Department of Global Health, Medicine and Welfare, Nagasaki University Graduate School of Biomedical \\ Sciences, Nagasaki, Japan
}

\begin{abstract}
Osteoporosis is a common disorder in aging populations that imposes considerable health problems. Tartrate-resistant acid phosphatase type $5 b$ (TRAP-5b) is derived from osteoclasts, and is involved in normal bone homeostasis. Recently, a novel assay system for TRAP-5b, the fragments absorbed immunocapture enzymatic assay method, has been developed. To evaluate the suitability of TRAP-5b as a screening marker for bone mineral density (BMD), we explored the correlations between serum TRAP-5b concentrations and laboratory findings, body mass index, or BMD in 462 community-dwelling elderly individuals (249 men and 213 women, age $73.4 \pm 6.5$ years) who participated in a regular medical screening program. By multivariate linear regression analysis adjusted for confounding factors, TRAP-5b was significantly correlated with body mass index $(\beta=-0.005, p=0.043)$, alkaline phosphatase, a marker for osteoid formation and calcification $(\beta=0.001, p<0.001)$, and triglyceride $(\beta=-0.097, p=0.016)$ in men, and with body mass index $(\beta=-0.009, p=0.025)$, alkaline phosphatase $(\beta=0.001, p<0.001)$, calcium $(\beta=-0.059, p=0.039)$, and bone trabecular area ratio $(\beta=-0.47, p=0.025)$ in women. In conclusion, the elevated serum level of TRAP- $5 b$ is independently correlated with the decreased BMD in women, but not in men. Because measurement of TRAP-5b is not affected by food intake, and blood samples can be collected at any time of the day, we suggest the suitability of serum TRAP- $5 \mathrm{~b}$ as a simple marker for the evaluation of BMD in women.
\end{abstract}

Keywords: bone mineral density; elderly; osteoporosis; screening; tartrate-resistant acid phosphatase type 5b Tohoku J. Exp. Med., 2011, 224 (2), 105-110. C 2011 Tohoku University Medical Press

Osteoporosis is a common disorder in aging populations that imposes considerable health problems. Because bone loss plays an important role in osteoporosis, evaluation of bone mineral density (BMD) plays an important role in its prevention (Kanis 2002; Qin et al. 2008; Yamauchi et al. 2010). However, changes in BMD are gradual and difficult to detect until substantial bone loss has occurred (Murano et al. 2003; Qin et al. 2008). Furthermore, BMD depends on the measurement sites (Hannan et al. 2000; Melton et al. 2000; Kanis 2002; Cui et al. 2005; Lenora et al. 2007; Qin et al. 2008; Basurto et al. 2009), such as femoral neck, lumbar spine, trochanter, total hip, radius, and calcaneus, as well as on bone densitometry equipment (Yamauchi et al. 2010).

Therefore, it would be valuable to develop effective and convenient biochemical markers for the screening for osteoporosis. Various biochemical markers, such as serum/ urine N-terminal cross-linked telopeptides of type I colla- gen (NTX), C-terminal cross-linked telopeptides of type I collagen (CTX), and deoxypyridinoline (DPD) as bone absorption markers, and bone alkaline phosphatase (BAP) and intact osteocalcin (OC) as bone formation markers, have been used as independent predictors of osteoporotic fracture risk and to evaluate the effect of osteoporosis treatment. However, measurements of these markers are relatively complicated and variability is still high, because osteoclastic activity is mainly assessed by measuring urinary markers (Ohashi et al. 2007).

Tartrate-resistant acid phosphatase (TRAP) has also been used as a bone resorption marker. Human serum contains two forms of TRAP, $5 \mathrm{a}$ and $5 \mathrm{~b}$; TRAP-5b is derived from osteoclasts and TRAP-5a from other, unidentified sources, probably activated macrophages (Hallen et al. 2002) and dendritic cells in chronic inflammation (Janckila and Yam 2009). Previously, Hallen et al. (2000, 2002) developed a novel immunoassay specific for $5 \mathrm{~b}$ using a

Received March 3, 2011; revision accepted for publication April 30, 2011. doi: 10.1620/tjem.224.105

Correspondence: Naomi Hayashida, M.D., Ph.D., Department of Global Health, Medicine and Welfare, Nagasaki University Graduate

School of Biomedical Science, 1-12-4 Sakamoto, Nagasaki 852-8523, Japan.

e-mail: naomin@nagasaki-u.ac.jp 
monoclonal antibody. Recently, Ohashi et al. (2007) developed a novel assay system for TRAP- $5 b$ called the fragments absorbed immunocapture enzymatic assay (FAICEA) method. Serum TRAP-5b activity has a low diurnal variability, its level is not affected by feeding, and it does not accumulate in the circulation in cases of renal or hepatic failure, so that the serum samples may be collected at any time of the day (Hallen et al. 2000, 2002; Ohashi et al. 2007; Nishizawa et al. 2008; Qin et al. 2008). Previous studies have shown that activity of serum TRAP-5b was significantly elevated in patients with osteoporosis and had a significant negative correlation with BMD (Halleen et al. 2002; Qin et al. 2008). Ohashi et al. (2007) reported that postmenopausal women had higher TRAP-5b concentrations than premenopausal women, but no significant differences among age groups were found in healthy men. However, there has been no previous evaluation of the suitability of TRAP-5b as a screening tool for BMD in community-dwelling elderly individuals.

In this study, we screened TRAP- $5 \mathrm{~b}$ concentrations in the general population of elderly individuals and clarified its correlation with laboratory findings and with BMD to evaluate the suitability of TRAP-5b as a screening marker for osteoporosis in the general population.

\section{Methods}

Subjects

Prior to the study, approval was obtained from the ethics committee of Nagasaki University (project registration number: 08122516). The study was conducted during a regular medical screening program for individuals more than 63 years old residing in Nagayo Town, Nagasaki Prefecture, Japan. After obtaining informed consent, we enrolled 462 Japanese participants (249 men and 213 women, age $73.4 \pm 6.5$ years) in this study.

\section{Measurement of bone trabecular area ratio}

Bone density was measured using an ultrasound bone densitometer (Benus $3^{\circledR}$, Ishikawa Seisakusyo, Ltd., Ishikawa, Japan). The densitometer was used to measure BMD of the calcaneus, based on the bone trabecular area ratio (BAR), as an index for BMD at other sites. Densitometry was based on fractal dimension analysis for bone area ratio and characterized by a coefficient of variation $(\mathrm{CV})$ of $1.6 \%$. The BAR refers to the proportion of bone tissue in a cross-section of the calcaneus bone, which is calculated from the ultrasound conduction velocity in that bone (Nohara et al. 2009).

\section{Data collection and laboratory measurement}

Height and weight were measured and body mass index (BMI; $\mathrm{kg} / \mathrm{m}^{2}$ ) was calculated as an index of obesity. Blood samples were collected from each participant after fasting overnight; intake of water was also prohibited. Aspartate aminotransferase (AST), alanine aminotransferase (ALT), $\gamma$-glutamyltranspeptidase $(\gamma$-GTP), total cholesterol (TC), triglyceride (TG), high density lipoprotein cholesterol (HDL-C), low density lipoprotein cholesterol (LDL-C), creatinine $(\mathrm{Cr})$, calcium $(\mathrm{Ca})$, iron $(\mathrm{Fe})$, alkaline phosphatase (ALP), red blood cells $(\mathrm{RBC})$, hemoglobin $(\mathrm{Hb})$, and hematocrit $(\mathrm{Ht})$ were measured by standard laboratory procedures. Serum TRAP-5b activity was measured by FAICEA (DS Pharma Biomedical Co. Ltd., Suita City, Japan). The normal range of TRAP-5b is $1.70-5.90 \mathrm{U} / 1$ in men and 2.50-7.60 U/1 in postmenopausal women.

\section{Statistical analysis}

Results are expressed as mean \pm standard deviation or median (25th-75th percentile) and differences between men and women were evaluated using the $t$-test or Mann-Whitney $U$ test. The correlations between TRAP-5b and BAR and other laboratory values were evaluated by univariate linear regression analysis and by multivariate linear regression analysis with adjustment. Because TG, HDL-C, AST, ALT, and $\gamma$-GTP levels were distributed in a skewed manner, logarithmic transformation was performed for the simple linear regression analysis and multivariate linear regression analysis. A probability value of less than 0.05 was considered to indicate statistical significance. All statistical analyses were performed using SPSS software, v. 17.0 for Windows (SPSS Japan, Tokyo, Japan).

\section{Results}

Characteristics of the study participants are shown in Table 1. Among the subjects analyzed, 61 women (28.6\%) and 43 men (17.3\%) were diagnosed as osteoporosis. Age was not significantly different between men and women ( $73.1 \pm 6.2$ years vs. $73.9 \pm 6.8$ years, $P=0.19)$. The BAR was significantly higher in men than in women $(28.6 \pm$ $3.4 \%$ vs. $27.0 \pm 3.1 \%, P<0.001)$ and TRAP- $5 \mathrm{~b}$ was significantly higher in women than in men $[4.0(3.1-4.9) \mathrm{U} / 1 \mathrm{vs.}$ 3.3 (2.6-4.1) U/1, $P<0.001]$. By univariate linear regression analysis, log TRAP-5b was significantly correlated with age $(r=0.28), \log \gamma$-GTP $(r=-0.24)$, ALP $(r=0.47)$, $\mathrm{Hb}(r=-0.23)$, BAR $(r=-0.20)$, BMI $(r=-0.17), \log \mathrm{TG}$ $(r=-0.15), \log$ AST $(r=-0.065), \log$ ALT $(r=-0.17), \mathrm{Ca}$ $(r=-0.14), \mathrm{Fe}(r=-0.21), \mathrm{RBC}(r=-0.20)$, and $\mathrm{Ht}(r=$ $-0.20)$ in men, and with BMI $(r=-0.27)$, BAR $(r=-0.20)$, ALP $(r=0.48), \log \mathrm{TG}(r=-0.16)$, and Ca $(r=-0.16)$ in women. In all participants, TRAP-5b was significantly correlated with age $(r=0.21)$, BMI $(r=-0.22)$, BAR $(r=$ $-0.25), \log$ TG $(r=-0.18), \log$ ALT $(r=-0.17), \log \gamma$-GTP $(r=-0.25), \mathrm{Cr}(r=-0.16), \mathrm{ALP}(r=0.47), \mathrm{RBC}$ $(r=-0.19), \mathrm{Hb}(r=-0.24)$, and $\mathrm{Ht}(r=-0.21)$ (Table 2).

By multivariate linear regression analysis adjusted for age, $\log$ TRAP-5b was significantly correlated with ALP $(\beta=0.001, P<0.001)$, BAR $(\beta=-0.007, P=0.032)$, BMI $(\beta=-0.006, P=0.033), \log \mathrm{TG}(\beta=-0.085, P=0.050)$, $\log$ ALT $(\beta=-0.11, P=0.034), \log \gamma$-GTP $(\beta=-0.10, P=$ $0.002), \mathrm{Fe}(\beta=-0.001, P=0.008), \mathrm{RBC}(\beta=0, P=0.043)$, $\mathrm{Hb}(\beta=-0.017, P=0.020)$, and $\mathrm{Ht}(\beta=-0.005, P=0.049)$ in men, and with ALP $(\beta=0.001, P<0.001), \operatorname{BAR}(\beta=$ $-0.009, P=0.014)$, BMI $(\beta=-0.011, P<0.001), \log$ TG $(\beta=-0.09, P=0.036), \log$ HDL-C $(\beta=0.21, P=0.049)$, $\mathrm{Cr}(\beta=-0.17, P=0.033)$, and $\mathrm{Ca}(\beta=-0.066, P=0.047)$ in women (Table 3). Furthermore, when multivariate linear regression analysis was performed by age and confounding factors, $\log$ TRAP-5b was significantly correlated with BMI $(\beta=-0.005, P=0.043)$, ALP $(\beta=0.001, P<0.001)$, and $\log$ TG $(\beta=-0.097, P=0.016)$ in men and with BMI $(\beta=$ $-0.009, P=0.001)$, ALP $(\beta=0.001, P<0.001)$, Ca $(\beta=$ 
Table 1. Characteristics of the study participants.

\begin{tabular}{|c|c|c|c|c|}
\hline Variables & Men $(n=249)$ & Women $(n=213)$ & All $(n=462)$ & $p$ Value \\
\hline Age (years) & $73.1 \pm 6.2$ & $73.9 \pm 6.8$ & $73.4 \pm 6.5$ & 0.19 \\
\hline BAR $(\%)$ & $28.6 \pm 3.4$ & $27.0 \pm 3.1$ & $27.9 \pm 3.3$ & $<0.001$ \\
\hline TRAP-5b (U/l) & $3.3(2.6-4.1)$ & $4.0(3.1-4.9)$ & $3.5(2.8-4.5)$ & $<0.001$ \\
\hline $\operatorname{BMI}\left(\mathrm{kg} / \mathrm{m}^{2}\right)$ & $24.1 \pm 3.0$ & $23.8 \pm 3.6$ & $23.9 \pm 3.5$ & 0.36 \\
\hline $\mathrm{TC}(\mathrm{g} / \mathrm{l})$ & $2.0 \pm 0.3$ & $2.1 \pm 0.3$ & $2.0 \pm 0.3$ & 0.016 \\
\hline TG $(g / l)$ & $1.2(0.9-1.8)$ & $1.0(0.7-1.6)$ & $1.1(0.8-1.7)$ & 0.008 \\
\hline HDL-C (g/l) & $0.54(0.50-0.60)$ & $0.60(0.50-0.70)$ & $0.57(0.50-0.70)$ & $<0.001$ \\
\hline LDL-C (g/l) & $1.2 \pm 0.3$ & $1.2 \pm 0.3$ & $1.2 \pm 0.3$ & 0.17 \\
\hline AST (U/l) & $24.0(20.0-28.1)$ & $23.0(19.0-26.0)$ & $23.0(20.0-27.0)$ & 0.006 \\
\hline $\operatorname{ALT}(\mathrm{U} / \mathrm{l})$ & $19.0(15.0-27.0)$ & $17.0(14.0-22.0)$ & $18.0(14.0-24.0)$ & $<0.001$ \\
\hline$\gamma$-GTP (U/l) & $29.0(20.0-51.0)$ & $18.0(13.0-25.0)$ & $22.0(16.0-35.3)$ & $<0.001$ \\
\hline $\mathrm{Cr}(\mathrm{mg} / \mathrm{l})$ & $8.4 \pm 1.9$ & $6.1 \pm 1.4$ & $7.3 \pm 2.0$ & $<0.001$ \\
\hline $\mathrm{Ca}(\mathrm{mg} / \mathrm{l})$ & $90.5 \pm 3.6$ & $91.3 \pm 3.4$ & $90.5 \pm 3.6$ & 0.023 \\
\hline $\mathrm{Fe}(\mathrm{mg} / \mathrm{l})$ & $1.1 \pm 0.4$ & $1.0 \pm 0.3$ & $1.0 \pm 0.3$ & $<0.001$ \\
\hline $\operatorname{ALP}(\mathrm{U} / \mathrm{l})$ & $250.0 \pm 75.3$ & $254.7 \pm 76.6$ & $252.2 \pm 75.9$ & 0.51 \\
\hline $\mathrm{RBC}\left(10^{10}\right.$ cells $\left./ \mathrm{l}\right)$ & $460.3 \pm 45.1$ & $426.8 \pm 41.2$ & $444.9 \pm 46.4$ & $<0.001$ \\
\hline $\mathrm{Hb}(\mathrm{g} / \mathrm{l})$ & $143.8 \pm 13.8$ & $129.6 \pm 11.4$ & $137.2 \pm 14.6$ & $<0.001$ \\
\hline Ht $(\%)$ & $44.4 \pm 3.8$ & $40.8 \pm 3.4$ & $42.7 \pm 4.0$ & $<0.001$ \\
\hline
\end{tabular}

Values are mean \pm standard deviation or median (25th-75th percentile)

Abbreviations: BAR, bone trabecular area rate; TRAP-5b, tartrate-resistant acid phosphatase 5b; BMI, body mass index; TC, total cholesterol; TG, triglyceride; HDL, high density lipoprotein cholesterol; LDL, low density lipoprotein cholesterol; AST, aspartate aminotransferase; ALT, alanine aminotransferase; $\gamma$-GTP, $\gamma$-glutamyltranspeptidase; Cr, creatinine; $\mathrm{Ca}$, calcium; Fe, iron; ALP, alkaline phosphatase; $\mathrm{RBC}$, red blood cells; Hb, hemoglobin; $\mathrm{Ht}$, hematocrit.

$p$ Value: Men vs. Women.

Table 2. Simple linear regression analysis of $\log$ TRAP-5b and other variables in men, in women, and all subjects.

\begin{tabular}{|c|c|c|c|c|c|c|}
\hline \multirow{2}{*}{ Variables } & \multicolumn{2}{|c|}{ Men } & \multicolumn{2}{|c|}{ Women } & \multicolumn{2}{|c|}{ ALL } \\
\hline & Correlation & $p$ Value & Correlation & $p$ Value & Correlation & $p$ Value \\
\hline Age & 0.28 & $<0.001$ & 0.11 & 0.10 & 0.21 & $<0.001$ \\
\hline BAR & -0.20 & 0.001 & -0.20 & 0.004 & -0.25 & $<0.001$ \\
\hline BMI & -0.17 & 0.008 & -0.27 & $<0.001$ & -0.22 & $<0.001$ \\
\hline $\mathrm{TC}$ & -0.087 & 0.17 & 0.002 & 0.97 & -0.017 & 0.72 \\
\hline $\log \mathrm{TG}$ & -0.15 & 0.019 & -0.16 & 0.021 & -0.18 & $<0.001$ \\
\hline $\log$ HDL-C & -0.017 & 0.79 & 0.13 & 0.066 & 0.079 & 0.090 \\
\hline LDL-C & -0.017 & 0.79 & 0.012 & 0.86 & 0.012 & 0.80 \\
\hline $\log \mathrm{AST}$ & -0.065 & 0.031 & -0.009 & 0.89 & 0.058 & 0.22 \\
\hline $\log$ ALT & -0.17 & 0.009 & -0.12 & 0.077 & -0.17 & $<0.001$ \\
\hline $\log \gamma$-GTP & -0.24 & $<0.001$ & -0.12 & 0.082 & -0.25 & $<0.001$ \\
\hline $\mathrm{Cr}$ & 0.007 & 0.92 & -0.12 & 0.081 & -0.16 & $<0.001$ \\
\hline $\mathrm{Ca}$ & -0.14 & 0.023 & -0.16 & 0.024 & -0.12 & 0.010 \\
\hline $\mathrm{Fe}$ & -0.21 & 0.001 & 0.013 & 0.85 & -0.15 & 0.001 \\
\hline ALP & 0.47 & $<0.001$ & 0.48 & $<0.001$ & 0.47 & $<0.001$ \\
\hline $\mathrm{RBC}$ & -0.20 & 0.002 & -0.013 & 0.85 & -0.19 & $<0.001$ \\
\hline $\mathrm{Hb}$ & -0.23 & $<0.001$ & -0.043 & 0.53 & -0.24 & $<0.001$ \\
\hline $\mathrm{Ht}$ & -0.20 & 0.001 & -0.012 & 0.87 & -0.21 & $<0.001$ \\
\hline
\end{tabular}

Abbreviations as in Table 1. 
Table 3. Multivariate linear regression analysis of $\log$ TRAP-5b adjusted by age in men and in women.

\begin{tabular}{|c|c|c|c|c|c|c|}
\hline \multirow{2}{*}{ Variables } & \multicolumn{3}{|c|}{ Men } & \multicolumn{3}{|c|}{ Women } \\
\hline & $\beta$ & $95 \% \mathrm{CI}$ & $p$ Value & $\beta$ & $95 \% \mathrm{CI}$ & $p$ Value \\
\hline BAR & -0.007 & $-0.012,-0.001$ & 0.032 & -0.009 & $-0.017,-0.002$ & 0.014 \\
\hline BMI & -0.006 & $-0.012,-0.001$ & 0.033 & -0.011 & $-0.017,-0.006$ & $<0.001$ \\
\hline $\mathrm{TC}$ & $<0.001$ & $-0.001,0$ & 0.27 & $<0.001$ & $0,0.001$ & 0.58 \\
\hline $\log \mathrm{TG}$ & -0.085 & $-0.17,0$ & 0.050 & -0.09 & $-0.18,-0.01$ & 0.036 \\
\hline $\log$ HDL-C & -0.016 & $-0.17,0.13$ & 0.84 & 0.21 & $0.001,0.41$ & 0.049 \\
\hline LDL-C & 0 & $-0.001,0.001$ & 0.93 & $<0.001$ & $-0.001,0.001$ & 0.55 \\
\hline $\log \mathrm{AST}$ & -0.096 & $-0.25,0.06$ & 0.23 & -0.008 & $-0.17,0.15$ & 0.93 \\
\hline $\log$ ALT & -0.11 & $-0.21,-0.01$ & 0.034 & -0.079 & $-0.19,0.04$ & 0.17 \\
\hline $\log \gamma$-GTP & -0.10 & $-0.16,-0.04$ & 0.002 & -0.063 & $-0.15,0.02$ & 0.16 \\
\hline $\mathrm{Cr}$ & -0.065 & $-0.17,0.04$ & 0.24 & -0.17 & $-0.33,-0.01$ & 0.033 \\
\hline $\mathrm{Ca}$ & -0.045 & $-0.098,0.008$ & 0.099 & -0.066 & $-0.13,-0.001$ & 0.047 \\
\hline $\mathrm{Fe}$ & -0.001 & $-0.001,0$ & 0.008 & $<0.001$ & $-0.001,0.001$ & 0.76 \\
\hline ALP & 0.001 & $0.001,0.001$ & $<0.001$ & 0.001 & $0.001,0.001$ & $<0.001$ \\
\hline $\mathrm{RBC}$ & 0 & $-0.001,0$ & 0.043 & $<0.001$ & $0,0.001$ & 0.60 \\
\hline $\mathrm{Hb}$ & -0.017 & $-0.032,-0.003$ & 0.020 & 0.001 & $-0.020,0.021$ & 0.95 \\
\hline $\mathrm{Ht}$ & -0.005 & $-0.011,0$ & 0.049 & 0.002 & $-0.005,0.008$ & 0.64 \\
\hline
\end{tabular}

Abbreviations as in Table 1.

$\beta$ : Standardized regression coefficient

CI: Confidence interval

Table 4. Multivariate linear regression analysis of TRAP-5b adjusted by age and confounding factors in men and in women.

\begin{tabular}{|c|c|c|c|c|c|c|}
\hline \multirow{2}{*}{ Variables } & \multicolumn{3}{|c|}{ Men } & \multicolumn{3}{|c|}{ Women } \\
\hline & $\beta$ & $95 \% \mathrm{CI}$ & $p$ Value & $\beta$ & $95 \% \mathrm{CI}$ & $p$ Value \\
\hline BAR & -0.20 & $-0.55,0.16$ & 0.28 & -0.47 & $-0.88,-0.06$ & 0.025 \\
\hline BMI & -0.005 & $-0.010,0$ & 0.043 & -0.009 & $-0.014,-0.004$ & 0.001 \\
\hline $\log \mathrm{TG}$ & -0.097 & $-0.18,-0.02$ & 0.016 & -0.058 & $-0.14,-0.02$ & 0.15 \\
\hline $\mathrm{Ca}$ & -0.006 & $-0.043,0.056$ & 0.80 & -0.059 & $-0.12,0.003$ & 0.039 \\
\hline ALP & 0.001 & $0.001,0.001$ & $<0.001$ & 0.001 & $0.001,0.001$ & $<0.001$ \\
\hline $\mathrm{Hb}$ & -0.013 & $-0.027,0$ & 0.055 & 0.004 & $-0.015,0.023$ & 0.65 \\
\hline
\end{tabular}

Abbreviations as in Table 1.

$\beta$ : Standardized regression coefficient

CI: Confidence interval

$-0.059, P=0.039)$, and $\operatorname{BAR}(\beta=-0.47, P=0.025)$ in women (Table 4).

\section{Discussion}

Many studies have been conducted to evaluate the usefulness of bone turnover markers as predictors of bone loss in conjunction with the evaluation of novel pharmaceutical drugs for osteoporosis (Gerdhem et al. 2004; Lenora et al. 2007; Nishizawa et al. 2008). NTX, CTX, and DPD have been considered to be typical bone resorption markers and bone alkaline phosphatase (BAP) and intact osteocalcin (OC) have been considered to be the main bone formation markers. However, urinary levels of DPD, NTX, and CTX need to be corrected by creatinine concentration to accurately assess bone resorption (Nishizawa et al. 2005, 2008;
Lenora et al. 2007; Qin et al. 2008) because of individual differences in renal clearance (Nishizawa et al. 2008).

Tartrate-resistant acid phosphatase is also a wellknown bone resorption marker, but it has not been widely used in clinical practice because of its relatively weak specificity for bone (Ohashi et al. 2007; Nishizawa et al. 2008; Janckila and Yam 2009). Human TRAP has two subtypes, TRAP-5a and TRAP-5b (Halleen et al. 2002). TRAP-5a contains sialic acid, which is not found in TRAP-5b. Halleen et al. $(2000,2002)$ developed TRAP with optimally purified antigenic properties and $\mathrm{pH}$ from human osteoclasts, which was identical to TRAP-5b and completely different from TRAP-5a, suggesting that TRAP-5b is derived from osteoclasts and TRAP-5a from other, unidentified sources, probably activated macrophages (Halleen et al. 
2002) and dendritic cells in chronic inflammation (Janckila and Yam 2009). This suggests that TRAP-5b may be a specific marker of osteoclast activity. However, conventional assay systems for TRAP-5b are generally not sensitive enough for clinical specimens and have a narrow range of linearity. The lack of sensitivity is probably caused by cross-reactivity of the monoclonal antibody with TRAP-5b and TRAP-5a (Ohashi et al. 2007). Recently, Ohashi et al. (2007) developed the FAICEA method for TRAP-5b that avoids interference from TRAP-5a. The FAICEA method uses a novel substance, 2-chloro-4-nitrophenyl phosphate (CNPP), which is much more specific for TRAP-5b at the appropriate $\mathrm{pH}$ (6.4-6.6). The FAICEA method also applies Trk62, a monoclonal antibody that is raised against ultrapure TRAP-5b derived from bone. Furthermore, TRAP-5b fragments are captured by Trk49, an absorbed monoclonal antibody with a high affinity for TRAP-5b fragments. The elimination of fragments using Trk49 in the assay system enables the measurement range to be extended. Of note, Trk62 has an affinity to TRAP-5b in the presence of reduced levels of competitor (Ohashi et al. 2007).

In our study, TRAP-5b was significantly correlated with BAR by univariate linear regression analysis in men $(r=-0.20)$ and women $(r=-0.20)$. Multivariate linear regression analysis adjusted for age also showed that TRAP-5b was significantly correlated with BAR in men $(\beta=-0.007, P=0.032)$ and women $(\beta=-0.009, P=0.014)$. When multivariate linear regression analysis was performed adjusted for confounding factors, TRAP-5b remained correlated with $\mathrm{BAR}$ in women $(\beta=-0.47, P=0.025)$ but not in men $(\beta=-0.20, P=0.28)$, which suggests that the suitability of TRAP-5b as a screening tool for BMD may be different between men and women. Estrogen deficiency associated with menopause is the primary cause of rapid bone loss in women. Nishizawa et al. (2008) reported that menopause was the signal of elevation of TRAP-5b activity and its activity remained at a high level even after menopause in women. Qin et al. (2008) and Nishizawa et al. (2008) also reported that the level of TRAP-5b was significantly higher in women with osteoporosis than in women with normal bone mass regardless of premenopausal or postmenopausal status. On the other hand, it is known that TRAP-5b varies little in elderly men with aging (Nishizawa et al. 2008). Different kinetics might cause discrepant results between the sexes and influence the suitability of TRAP-5b as a screening tool. Another possibility is the different contribution of muscle mass between the sexes. Fujiwara et al. (2005) reported that individuals with high fracture risk were more likely to be older, lighter, shorter, and to have lower BAR, which suggested that higher values of muscle mass were associated with increased BMD. The relatively greater contribution of muscle mass to BMD may also cause discrepant results.

Our results showed that TRACP-5b was independently correlated with BMI both in men and women. Barrera et al. (2004) suggested that BMI is the best independent predictor of BMD in women and in men. Tang et al. (2007) found that BMI was independently and positively associated with broadband ultrasound attenuation in older men. As shown in a previous study, appropriate maintenance of BMI is important to prevent the loss of bone mass that occurs after menopause (Crepaldi et al. 2007).

By multivariate regression analysis adjusted for confounding factors, we showed that TRAP-5b was positively correlated with ALP both in men $(\beta=0.001, P<0.001)$ and in women $(\beta=0.001, P<0.001)$. We also showed that TRAP-5b was negatively correlated with $\mathrm{Ca}$ in women $(\beta=$ $-0.059, P=0.039)$. Because it is well known that ALP is a classical marker for osteoid formation and calcification, and that $\mathrm{Ca}$ is important to maintain bone density and is lost with aging especially in women (Hannan et al. 2000; Izumotani et al. 2003; Meier et al. 2005; Lanham-New 2008; Hien et al. 2008), our present results suggest that TRAP-5b is a sensitive marker for screening for BMD in the general population of elderly individuals, especially women.

There are several limitations in this study. We could not exclude participants who had experienced steroid therapy, which affects BMD. We were also unable to perform bone histomorphometry, which has been accepted as the gold standard for characterizing the state of disease progression. Moreover, our study population may not have been sufficiently large, although we studied the available community-dwelling elderly individuals who participated in a regular medical screening program once a year. Further studies are needed to clarify the availability of TRAP-5b for screening of BMD.

In conclusion, we screened TRAP-5b in communitydwelling elderly individuals and found that TRAP-5b was independently correlated with BMD in women, but not in men. Because measurement of TRAP-5b is not affected by food intake and blood samples could be collected at any time of the day, we suggest the suitability of TRAP-5b as a simple screening tool for the evaluation of BMD, especially in women. Further studies are needed to develop a comprehensive strategy for screening for osteoporosis in the general elderly population.

\section{Acknowledgments}

This work was supported by the Ministry of Education, Culture, Sports, Science, and Technology of Japan through the Nagasaki University Global Center of Excellence (COE) program. We would like to thank Taihei Ishikawa, Taiyo Jinno, Yuichiro Nakamura, Haruhi Furukawa, Miho Yoshida, Satomi Nagamoto, Momoko Yamaguchi, Kyoko Hagane, Makiko Orita, and Aiko Ienaga for their technical assistance.

\section{Conflict of Interest}

The authors declare no conflict of interest.

\section{References}

Barrera, G., Bunout, D., Gattás, V., de la Maza, M.P., Leiva, L. \& Hirsch, S. (2004) A high body mass index protects against 
femoral neck osteoporosis in healthy elderly subjects. Nutrition, 20, 769-771.

Basurto, L., Galván, R., Cordova, N., Saucedo, R., Vargas, C., Campos, S., Halley, E., Avelar, F. \& Zárate, A. (2009) Adiponectin is associated with low bone mineral density in elderly men. Eur. J. Endocrinol., 160, 289-293.

Crepaldi, G., Romanato, G., Tonin, P. \& Maggi, S. (2007) Osteoporosis and body composition. J. Endocrinol. Invest., 30, 42-47.

Cui, L.H., Shin, M.H., Chung, E.K., Lee, Y.H., Kweon, S.S., Park, K.S. \& Choi, J.S. (2005) Association between bone mineral densities and serum lipid profiles of pre- and post-menopausal rural women in South Korea. Osteoporos. Int., 16, 1975-1981.

Fujiwara, S., Sone, T., Yamazaki, K., Yoshimura, N., Nakatsuka, K., Masunari, N., Fujita, S., Kushida, K. \& Fukunaga, M. (2005) Heel bone ultrasound predicts non-spine fracture in Japanese men and women. Osteoporos. Int., 16, 2107-2112.

Gerdhem, P., Ivaska, K.K., Alatalo, S.L., Hallen, J.M., Hellman, J., Isaksson, A., Pettersson, K., Väänänen, H.K., Åkesson, K. \& Obrant, K.J. (2004) Biochemical markers of bone metabolism and prediction of fracture in elderly women. J. Bone Miner. Res., 19, 386-393.

Halleen, J.M., Alatalo, S.L., Suominen, H., Cheng, S., Janckila, A.J. \& Väänänen, H.K. (2000) Tartrate-resistant acid phosphatase $5 \mathrm{~b}$ : a novel serum marker of bone resorption. J. Bone Miner. Res., 15, 1337-1345.

Halleen, J.M., Ylipahkala, H., Alataro, S.L., Janckila, A.J., Heikkinen, J.E., Suominen, H., Cheng, S. \& Väänänen, H.K. (2002) Serum tartrate-resistant acid phosphatase $5 \mathrm{~b}$, but not $5 \mathrm{a}$, correlates with other markers of bone turnover and bone mineral density. Calcif. Tissue Int., 71, 20-25.

Hannan, M.T., Felson, D.T., Dawson-Hughes, B., Tucker, K.L., Cupples, L.A., Wilson, P.W. \& Kiel, D.P. (2000) Risk factors for longitudinal bone loss in elderly men and women: The Framingham Osteoporosis Study. J. Bone Miner. Res., 15, 710-720.

Hien, V.T.T., Khan, N.C., Mai, Le.B., Lam, N.T., Phuong, T.M., Nhung, B.T., Nhien, N.V., Nakamori, M. \& Yamamoto, S. (2008) Effect of community-based nutrition education intervention on calcium intake and bone mass in postmenopausal Vietnamese women. Public Health Nutr., 12, 674-679.

Izumotani, K., Hagiwara, S., Izumotani, T., Miki, T., Morii, H. \& Nishizawa, Y. (2003) Risk factors for osteoporosis in men. $J$. Bone Miner. Metab., 21, 86-90.

Janckila, A.J. \& Yam, L.T. (2009) Biology and clinical significance of tartrate-resistant acid phosphatases: new perspectives on an old enzyme. Calcif. Tissue Int., 85, 465-483.

Kanis, J.A. (2002) Diagnosis of osteoporosis and assessment of fracture risk. Lancet, 359, 1929-1936.

Lanham-New, S.A. (2008) Importance of calcium, vitamin D. and vitamin $\mathrm{K}$ for osteoporosis prevention and treatment. Proc. Nutr. Soc., 67, 163-176.

Lenora, J., Ivaska, K.K., Obrant, K.J. \& Gerdhem, P. (2007) Prediction of bone loss using biochemical markers of bone turnover. Osteoporos. Int., 18, 1297-1305.

Meier, C., Nguyen, T.V., Center, J.R., Seibel, M.J. \& Eisman, J.A. (2005) Bone resorption and osteoporotic fractures in elderly men: the Dubbo osteoporosis epidemiology study. J. Bone Miner. Res., 20, 579-587.

Melton, L.J. 3rd, Khosla, S., Atkinson, E.J., Oconnor, M.K., Ofallon, W.M. \& Riggs, B.L. (2000) Cross-sectional versus longitudinal evaluation of bone loss in men and women. Osteoporos. Int., 11, 592-599.

Murano, T., Izumi, S.I., Kika, G., Haque, S.F., Okuwaki, S., Mori, A., Suzuki, T., Matsubayashi, H., Ikeda, M., Goya, K. \& Makino, T. (2003) Impact of menopause on lipid and bone metabolism and effect of hormone replacement therapy. Tokai J. Exp. Clin. Med., 28, 109-119.

Nishizawa, Y., Inaba, M., Ishii, M., Yamashita, H., Miki, T., Goto, H., Yamada, S., Chaki, O., Kurasawa, K. \& Mochizuki, Y. (2008) Reference intervals of serum tartrate-resistant acid phosphatase type $5 \mathrm{~b}$ activity measured with a novel assay in Japanese subjects. J. Bone Miner. Metab., 26, 265-270.

Nishizawa, Y., Nakamura, T., Ohta, H., Kushida, K., Gorai, I., Shiraki, M., Fukunaga, M., Hosoi, T., Miki, T., Chaki, O., Ichimura, S., Nakatsuka, K. \& Miura, M. (2005) Guidelines for the use of biochemical markers of bone turnover in osteoporosis (2004). J. Bone Miner. Metab., 23, 97-104.

Nohara, T., Ueda, M., Ohta, A. \& Sugimoto, T. (2009) Correlation of body growth and bone mineral density measured by ultrasound densitometry of the calcaneus in children and adolescents. Tohoku J. Exp. Med., 219, 63-69.

Ohashi, T., Igarashi, Y., Mochizuki, Y., Miura, T., Inaba, N., Katayama, K., Tomonaga, T. \& Nomura, F. (2007) Development of a novel fragments absorbed immunocapture enzyme assay system for tartrate-resistant acid phosphatase 5b. Clin. Chim. Acta, 376, 205-212.

Qin, Y.J., Zhang, Z.L., Zhang, H., Hu, W.W., Liu, Y.J., Hu, Y.Q., Li, M., Gu, J.M. \& He, J.W. (2008) Age-related changes of serum tartrate-resistant acid phosphatase $5 \mathrm{~b}$ and the relationship with bone mineral density in Chinese women. Acta. Pharmacol. Sin., 29, 1493-1498.

Tang, Y.J., Sheu, W.H., Liu, P.H., Lee, W.J. \& Chen, Y.T. (2007) Positive associations of bone mineral density with body mass index, physical activity, and blood triglyceride level in men over 70 years old: a TCVGHAGE study. J. Bone Miner. Metab., 25, 54-59.

Yamauchi, H., Fukunaga, M., Nishikawa, A. \& Orimo, H. (2010) Changes in distribution of bone densitometry equipment from 1996 to 2006 in Japan. J. Bone Miner. Metab., 28, 60-67. 